\title{
An Instruction for Direct Interpretation of LZ77-compressed Programs
}

\author{
Christopher W. Fraser \\ Microsoft Research \\ One Microsoft Way, Redmond, WA 98052, USA \\ cwfraser@microsoft.com
}

\section{Summary}

A new instruction adapts LZ77 compression for use inside running programs. The instruction economically references and reuses code fragments that are too small to package as conventional subroutines. The compressed code is interpreted directly, with neither prior nor on-the-fly decompression. Hardware implementations seem plausible and could benefit both memoryconstrained and more conventional systems.

The method is extremely simple. It has been added to a pre-existing, bytecoded instruction set, and it added only ten lines of $\mathrm{C}$ to the bytecode interpreter. It typically cuts code size by a third; that is, typical compression ratios are roughly $0.67 \mathrm{x}$. More ambitious compressors are available, but they are more complex, which retards adoption. The current method offers a useful trade-off to these more complex systems.

Keywords: bytecode, compression, interpreters.

\section{Introduction}

Code compression can be used to save network, disk, memory, and even cache space. The various scenarios require different techniques. Modem bottlenecks, for example, can justify compressors and decompressors too costly for use with disk compression software.

At the other end of the spectrum is directly interpretable code, either machine code or interpretive code. For these systems, the scarcity is at the lowest levels of the memory hierarchy, so there is no room to decompress the code at all. The choices are either decompression into an internal hardware instruction cache, queue, or buffer $[1,2,3,4,5,6,7]$ or interpretation without decompression $[8,9,10]$.

These constraints are less common on current personal computers or servers than on embedded systems such as cellular phones, where market pressures require maximizing features while minimizing ROM. Features that don't need the fastest responses — such as those associated with key presses—are candidates for optimizations that trade time for space, including interpretation.

This paper augments the toolbox for this scenario. Its method compresses a bytecode by about one third (that is, a compression ratio of $0.67 \mathrm{x}$ ), but it adds only ten lines of $C$ code $^{1}$ to the bytecode interpreter. It starts with a well-studied method from general-purpose data compression and adapts the method to the problem of compressing a program that must be interpreted directly, without decompression.

\section{Background: LZ77 compression}

LZ77 compression [11] accepts a stream of characters—typically bytes—and produces a stream that interleaves literals and pointers. Each pointer indicates a phrase in the preceding characters and has two parts: a displacement and a length. The displacement gives the distance back to the phrase, and the length identifies the number of characters in the phrase. For example, the byte string

Blah blah.

\footnotetext{
${ }^{1}$ Appendix 1 gives the $\mathrm{C}$ code.
} 
compresses to

Blah b $\underline{5,3}$.

where the underlined material denotes a pointer. The displacement is five, and the length is three, because the next three bytes repeat those back five bytes.

The actual encoding must distinguish pointers from literals, and there are a variety of ways to do so [12]. One method inserts bit masks. For example, an encoding of the compressed sequence above might use these bytes:

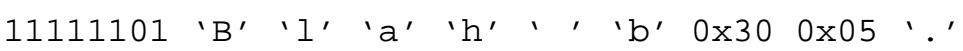

The initial byte above is a bit mask and displayed as such above; it reports the fact that there are six leading literal bytes ("Blah b"), followed by one pointer, followed by one last literal byte (the period). The literal bytes and pointer components follow the bit mask immediately, followed by the next bit mask, and so on. In the example above, a twelve-bit displacement (००5) follows a four-bit length (3).

More elaborate encodings offer multiple length or pointer sizes and thus widen the fields in the bit mask above to two or three bits [13]. These fields are thus roughly analogous to an instruction opcode, and the length, displacement, and literal data-though separated somewhat from the mask-are roughly analogous to instruction operand fields. LZ decompressors interpret these "instructions" in a single pass over the input; the method presented below interprets them as part of an interpreter for conventional instructions.

\section{The Echo instruction}

The current work adds LZ77 pointers to conventional instruction sets. The assembler instruction

echo $.-5,3$

commands the (hardware or software) interpreter to fetch and execute three instructions starting five bytes back from the echo instruction. The assembler encoding suggested above is simply a readable version of the binary encoding seen by the interpreter. Some readers might find it helpful to think of echo instructions as like C's \#include directives but with the substitution happening dynamically in an instruction cache or buffer.

In pseudo-code, the semantics for an echo instruction are simple:

1. Save the PC.

2. Subtract the contents of the echo instruction's displacement field from the PC.

3. Set $\mathrm{N}$ to the contents of the echo instruction's length field.

4. Fetch and execute the instruction at the address in the PC.

5. Decrement $\mathrm{N}$ and go back to Step 4 if the result exceeds zero.

6. Restore the PC and bump it past the echo instruction.

Some echo instructions will point back to phrases that include other echo instructions. This feature obliges the interpreter to maintain a stack of program counters and lengths. As the interpreter bumps the former, it subtracts one from the latter. When a length drops to zero, the interpreter pops the stack and thus resumes interpretation of the containing echo instruction. The stack is no problem for software interpreters, but hardware implementations might benefit from a small upper bound on the nesting level, which could be enforced easily by compilers or post-processors that create the echo instructions.

Compression improves if echo instructions can also reference fragments of the streams represented by earlier echo instructions. Consider this example:

$$
\begin{aligned}
& \text { echo } .-100,4 \\
& \text { echo } .-200,4
\end{aligned}
$$

and assume that these two echo instructions reference only primitive or base instructions, not other echo instructions. Suppose now that the program later repeats the last seven of the eight base instructions. This seven-instruction phrase might not appear anywhere in the compressed program, because the two four-instruction phrases above aren't adjacent. Such a program would benefit from an extended echo instruction, which appends an additional field that tells how many leading primitive (that is, non-echo) instructions to skip. For example, 
echo $.-10,2,1$

interprets two instructions located back ten bytes—namely, the two previous echo instructions—but the interpreter skips the first primitive (that is, non-echo) instruction yielded by the instruction phrase, which is always guaranteed to start with another echo instruction. When it is necessary to distinguish between echo instructions with and without offset fields, the qualifiers "extended" and "basic" are used below. Extended echo instructions destroy the rough similarity that basic echo instructions have to $\mathrm{C} \#$ include directives, because the latter cannot skip part of an inclusion.

The semantics above for echo instructions work for straight-line code but fail for phrases with internal control flow, for at least two reasons. First, the static instruction count in an echo instruction could get out of sync with the number of instructions executed dynamically. Suppose, for example, that a program has exactly one repeated phrase, which includes a conditional branch over one or more instructions also within the phrase. Then an execution of the echo instruction could take the branch and cause the loop above to execute unwanted instructions past the end of the first instance of the phrase.

There is a second failure mode for control flow within echoed phrases: Step 6 above could quash valid jumps. Suppose, for example, that the PC is changed by an unconditional jump executed by Step 4 above. Suppose also that $\mathrm{N}$ happens to be one at this point, so the loop is about to exit. Then Step 6 clobbers the new value in the PC before it can be used, nullifying the jump before it can take effect.

Implementations may solve these problems by forbidding echo instructions to point back to phrases that embed control transfers. That is, when recognizing a repeated phrase, the compressor must truncate the extension of the phrase when the next operator is found to be a branch or jump. A more complicated semantics might avoid this problem by, for example, exiting all nested echo instructions at jumps.

The implementation used here invokes a fresh copy of the interpreter (with its own PC) at each call, so it is not obliged to treat calls and returns like branches and jumps and thus allows echo instructions to reference phrases with calls and returns. Alternative treatments of calls and returns are considered below.

Labels present another complication. If the phrase referenced by an echo instruction spans a label, then jumps to such labels would need to skip the phrase elements before that label. In order to meet this requirement, such jumps would need to be augmented with a field encoding this offset, and such fields would naturally hurt compression. Worse, indirect jumps would need either a distinct offset field for each potential target or a restriction that all targets share a common offset. To avoid these complications, echo instructions have to date been constrained to reference no labels. That is, the LZ compressor stops extending a phrase when it encounters a label.

The echo instruction complements typical call or subroutine-jump instructions. Both allow reuse of common code fragments, but they make different trade-offs and thus benefit different situations. Table 1 contrasts the trade-offs. A key difference for compression is that conventional subroutines — even light-weight ones [14, 15, 16]—need a return instruction, which makes some small fragments uneconomical and precludes flowing or falling into the first copy of each fragment.

Table 1. Call versus Echo

\begin{tabular}{|l|l|}
\hline Call instructions: & Echo instructions: \\
\hline $\begin{array}{l}\text { Use subroutine prologues and epilogues, which add at least } \\
\text { one instruction, namely a return. }\end{array}$ & $\begin{array}{l}\text { Omit prologues and epilogues, allowing reuse of fragments } \\
\text { too small to pay for the overhead of prologues and epilogues. }\end{array}$ \\
\hline Must replace each copy of the fragment with a call. & Can flow or fall into the first copy for free. \\
\hline $\begin{array}{l}\text { Delimit fragments in the callee, namely with the prologue } \\
\text { and epilogue. }\end{array}$ & $\begin{array}{l}\text { Delimit fragments in the caller, namely in the echo } \\
\text { instruction. }\end{array}$ \\
\hline Save state in visible registers and memory. & $\begin{array}{l}\text { Save state only in invisible registers, namely the stack of } \\
\text { active echo instructions. }\end{array}$ \\
\hline Permit arbitrary internal control flow and labels. & Forbid internal control flow and labels. \\
\hline
\end{tabular}




\section{The initial bytecode}

The initial implementation of echo instructions was built on a pre-existing bytecode interpreter. Its bytecode is a simple postfix encoding of lcc trees [17] and nearly identical to that used in a recent paper [9] on a very different, grammar-based method for bytecode compression.

Most operators consist of an un-typed or generic base (such as ADD) followed by a one-character type suffix (I for integer, $F$ for float, etc), which indicates the type of value produced. Appendix 2 lists all of the operators that appear in the bytecode. There are 99 valid operator-suffix pairs, leaving 256-99=157 codes for use in echo instructions. Allocating unused opcodes to echo instructions complicates fair comparisons with the original, uncompressed code, but compression experiments below estimate the separate contributions of the echo instruction and the extra opcodes.

All operators are encoded by a single byte, but a few are followed by one or more literal bytes. For example, LIT2 is followed by a two-byte constant, which it simply pushes onto the stack. Branch offsets and global addresses aren't known until after compression, so they are encoded using one level of indirection. That is, the instruction stream includes not the actual address but rather a two-byte literal index into a table that holds the actual address.

The representation has two other elements, namely procedure descriptors and trampolines for inter-operation with existing libraries and conventional, non-bytecoded procedures. These elements are not bytecoded and thus not subject to compression with echo instructions, so they are described elsewhere [9]. Only two material changes were made to this previous representation. First, the bytecode was formerly separated by procedure but has now been concatenated into one long string, in order to allow echo instructions to reach back and reuse bytecode from other procedures. Second, the previous grammarbased compression has been replaced with compression using echo instructions.

\section{The compressor}

The compressor accepts a program in this bytecode and emits an equivalent program in which echo instructions replace repeated phrases. This compressor changes branch offsets and needs to see all labels, so it naturally operates on an assemblycode representation of the bytecode, which uses symbolic labels. After compression, the code is "assembled" into a binary format for execution, though the assembly process is so simple that is accomplished by $\mathrm{C}$ macros.

This compressor could adapt any of the methods used by LZ compressors, which range from linear search within the window reachable by the widest displacement, to hash tables, to Patricia trees [12]. This work focuses on compression ratios, not compressor speed, so it uses a simple method that is easily modified during experimentation. Namely, the compressor maintains a hash table that maps each operator to a list that holds the address of each of the last twenty occurrences of said operator. This limit reduced compression for typical programs only trivially, but it improved compression time significantly.

When the compressor is at position $\mathrm{P}$, it compares the sequence of instructions at $\mathrm{P}$ with the sequences at each of these twenty earlier positions. The longest match is the winning phrase. If the echo instruction that encodes that phrase is shorter than the phrase itself, then the compressor emits the echo instruction and skips forward to the end of the phrase. Otherwise, it emits the instruction at $\mathrm{P}$ and skips forward by one instruction.

An echo instruction can pay for even one-instruction phrases, if the original instruction includes literal bytes, and if the echo instruction is short enough. For this reason, careful allocation of bytecodes to echo instructions is useful.

\section{Encoding echo instructions}

Adding echo instructions to an instruction set requires choosing an encoding. One approach might use Tunstall coding [18] or perhaps Huffman coding [19] constrained to emit codes with lengths that are multiples of eight bits. The results, however, would make it impossible to separate the effect of the Tunstall or Huffman coding from the effect of the echo instruction. Tunstall and Huffman coding have thus been saved for future work, and a simple hand-designed echo instruction is used below.

Since the uncompressed bytecode has only 99 distinct operators, it is possible to allocate bytecodes 128-255 to specialized echo instructions and still have a few escape codes left over for longer variations. For example, bytecodes 128-255 might be treated as a seven-bit instruction for phrases that can be referenced with a two-bit length and a five-bit displacement.

Competing for the scarce bytecodes are the length and displacement fields, the offset field for extended echo instructions, and the opcodes themselves. To guide the choice for the bytecoded instruction set above, some measurements were taken. The initial round of measurements focused on sizing the length and displacement fields and thus ignored the offset field for 
extended echo instructions. The bytecode for lcc itself was compiled and compressed assuming—naively—that the first byte might be able hold the opcode, length, and the first seven bits of displacement, and that the opcode could specify whether the displacement added 1, 2, 3, or 4 bytes.

Figure 1 shows the distribution of phrase lengths. Short phrases far outnumber longer ones, so the frequency axis uses a log scale. A 3-bit length field could handle values 1-8, which could handle $99 \%$ of the phrases in this sample, a 2-bit field could handle $95 \%$, and a 1-bit field could handle $81 \%$. Even a 0-bit field — that is, an echo instruction specialized to phrases of length 1 -could handle $54 \%$.

Figure 2 shows the distribution of the widths of displacements. Perhaps surprisingly, one-byte displacements can capture most of the benefit, but it makes sense to allow longer variations as well.

The initial round of measurements, which is summarized in Figures 1 and 2, suggests trying length fields of 0-3 bits and displacements ranging up to 13 bits. It suggests offering short encodings for length fields as narrow as zero bits and displacements as narrow as 4 bits. Thus the input was recompressed using the trial encodings below:

1. Use bytecodes 128-255 for echo instructions with length of one (that is, a zero-bit length field) and a displacement that fits in seven bits. When this won't do, use a three-byte form, composed of a one-byte opcode plus two literal bytes that hold a three-bit length and a 13-bit displacement.

2. As above, but the one-byte encodings use a one-bit length and a six-bit displacement.

3. As above, but the one-byte encodings use a two-bit length and a five-bit displacement.

4. Use bytecodes 128-255 plus one literal byte to encode a three-bit length and a twelve-bit displacement.

All trials above share a fully general nine-byte echo instruction (a one-byte opcode, two-byte length, two-byte offset, and four-byte displacement) for those few cases with a length or displacement that won't fit in the shorter encodings. Table 2 shows the results of these trial encodings on lcc itself. Encoding 2 wins and is thus used below, but the other encodings do nearly as well and offer plausible alternatives.

Table 2. Encoding trials

\begin{tabular}{|r|r|l|}
\hline Size & Ratio & Encoding \\
\hline 199,873 & 1.000 & Uncompressed \\
\hline 131,374 & 0.657 & Encoding 1: zero-bit lengths \\
\hline 127,385 & 0.637 & Encoding 2: one-bit lengths \\
\hline 131,581 & 0.658 & Encoding 3: two-bit lengths \\
\hline 129,816 & 0.649 & Encoding 4: no one-byte forms \\
\hline
\end{tabular}

\section{Effects of various constraints}

The current implementation of echo compression exploits a large number of free bytecodes and a fully recursive software interpreter. Other environments, particularly hardware implementations, might be more constrained, so three experiments were run to estimate the effect of several common constraints on echo compression.

First, lcc has many unused bytecodes, but other base instruction sets might be less obliging. In order to quantify the benefit of the spare bytecodes, lcc was recompressed using a variation on Encoding 2 that drops the one-byte echo instructions and replaces them with two-byte encodings. This variation uses only three of the 256 total bytecodes for echo instructions. The three echo instruction formats are: a two-byte form with an opcode plus one byte with one bit for the width and seven for the displacement; the three-byte form shared by Encodings 1-3 above; and the general, nine-byte form above. This fifth encoding compresses lcc to 136,968 bytes, for a compression ratio of $0.685 x$. Exploiting lcc's unused bytecodes saves another factor of $127,385 / 136,968$ or $.930 x$. The unused bytecodes thus help, but they contribute much less than simple echo compression. 
Second, some implementations might need to forbid all control flow within echoed phrases. The current implementation forbids conditional branches, unconditional jumps, and labels, but it permits calls and returns. If these too are forbidden, echo compression for lcc worsens slightly, from 127,385 bytes and a compression ratio of $0.637 \mathrm{x}$ to 131,521 bytes and a ratio of $0.658 x$. Elimination of all control flow with echo instructions thus imposed only a modest cost.

Finally, bytecode interpreters can easily support nested echo instructions via recursion, but some hardware implementations may need to limit or even forgo recursion. Table 3 illustrates the cost of such limitations by quantifying the compression of lcc using Encoding 2 and various nesting limits. When nesting is forbidden, the compression ratio worsens from the $0.637 \mathrm{x}$ above to $0.754 \mathrm{x}$, but a small, fixed-size stack does almost as well as the unconstrained interpreter. The code in Appendix 1 shows that only a few bytes of data need to be saved per nesting level.

Table 3. Nesting limits

\begin{tabular}{|r|r|l|}
\hline Size & Ratio & Nesting limit \\
\hline 150,636 & 0.754 & 0 \\
\hline 141,177 & 0.706 & 1 \\
\hline 136,180 & 0.681 & 2 \\
\hline 133,547 & 0.668 & 3 \\
\hline
\end{tabular}

\section{Encoding extended echo instructions}

Now for the extended echo instructions, which point back to another echo instruction and add an offset field that tells where the current phrase starts in the previous phrase. That is, the offset field specifies the number of primitive (that is, non-echo instructions) to skip from the earlier phrase. In order to size the offset field, lcc was recompiled using Encoding 4 above and with the (naïve) assumption that the extra field would require no more bits. Figure 3 shows the resulting distribution of offsets. Short offsets predominate, so the frequency uses a log scale. Offset zero is included to account for the frequencies of the basic echo instruction.

For the data in Figure 3, zero offsets outnumber the next most common offset by a factor of seven, so the basic echo instructions (that is, those with zero offsets) should keep the shorter encodings assigned above, and the extended echo instructions should use a longer encoding. Three bytes should do, because over $99 \%$ of the non-zero offsets fit in a four-bit field. Opcodes 100-115 can be used to encode both the operator and the offset, and two literal bytes can encode the three-bit length and a thirteen-bit displacement.

\section{Performance}

Echo instructions are very common in the compressed code. When lcc is compressed using the encodings defined above, the echo opcodes are the most common and account for roughly $20 \%$ of the instructions in the final program.

Table 4 gives the compression performance on a set of benchmarks for echo instructions using Encoding 2 above. The set includes all of the C integer benchmarks in the SPEC2000 set except for Perl, which uses nonstandard C features that lcc does not compile. Table 4 also includes $l c c$ itself plus three smaller programs—a solution to the eight queens problem, copt, and iburg - because smaller inputs give LZ compressors less time to get up to speed and thus can present challenges. Unlike conventional compiler benchmarks, which must vary such factors as instruction mix, program size, and demand for working set and instruction and data cache, benchmarks for simple code compression need mainly to vary program size and to avoid replicated code. It is important to vary program size because small programs can be hard to compress, and because large inputs are needed to test large phrase displacements. copt is a rule-based peephole optimizer for assembly code, and the rest of the benchmarks in the table should be familiar to those experienced with compilers. lcc's automatically generated code generators were removed because they could artificially inflate compression performance.

The comparison with gzip [20], which also uses LZ compression, unfairly favors gzip, which does not support direct interpretation and thus is free to include control flow in phrases and free to Huffman-code both literals and pointers, since it uses front-to-back decompression and need not support the byte-addressability commonly assumed by bytecode interpreters. 
Table 4. Compression performance

\begin{tabular}{|r|r|r|r|r|r|}
\hline & no compression & \multicolumn{2}{|c|}{ compressed with echo } & \multicolumn{2}{|c|}{ compressed with gzip } \\
\cline { 2 - 6 } & bytes & bytes & ratio & bytes & ratio \\
\hline 8queens & 439 & 262 & 0.597 & 195 & 0.444 \\
\hline copt & 2,907 & 1,993 & 0.686 & 1,151 & 0.396 \\
\hline mcf & 11,875 & 7,501 & 0.632 & 3,851 & 0.324 \\
\hline iburg & 12,544 & 8,328 & 0.664 & 4,361 & 0.348 \\
\hline bzip2 & 28,012 & 17,292 & 0.617 & 9,139 & 0.326 \\
\hline gzip & 47,178 & 30,595 & 0.649 & 15,977 & 0.339 \\
\hline parser & 96,115 & 60,383 & 0.628 & 26,780 & 0.279 \\
\hline vpr & 123,124 & 75,835 & 0.616 & 35,266 & 0.286 \\
\hline crafty & 190,222 & 117,177 & 0.616 & 54,826 & 0.288 \\
\hline lcc & 199,873 & 127,385 & 0.637 & 62,740 & 0.314 \\
\hline twolf & 225,177 & 136,644 & 0.607 & 61,318 & 0.272 \\
\hline vortex & 466,217 & 290,540 & 0.623 & 120,396 & 0.258 \\
\hline gap & 620,618 & 377,905 & 0.609 & 154,508 & 0.653 \\
\hline gcc & $1,425,390$ & 930,907 & 450,384 & 0.316 \\
\hline
\end{tabular}

One compressor in the related work [9] uses the same bytecode and has the same constraint for direct interpretation without decompression. It thus presents an unique opportunity for direct, fair comparison that is unavailable for the other related works below, at least not without access to or reimplementation of their code. This previous compressor [9] yielded compression ratios slightly better than gzip's, which means that the echo instruction achieves roughly half of what might be achieved in a directly interpretable bytecode. The previous compressor was, however much more complex, and its interpreter required two levels of interpretation or a much larger interpreter. The echo instruction thus presents a useful alternative. It offers non-trivial compression at a very low cost to the interpreter, where the previous compressor competes with the highlytuned and less constrained gzip, but at the cost of an interpreter that is significantly slower or larger.

\section{Other implementations}

Following the preliminary version of this paper [21], others have modified the technique for interpretation by hardware [22, 23, 24]. All report useful savings, but most compression ratios are lower, at least partly because material such as register numbers make machine code more prone to mismatches than bytecode.

Lau et al [22] target the Alpha architecture using the SimpleScalar simulator [25]. They add a new "bit-mask" echo, which includes a mask to skip execution of unwanted instructions from the earlier sequence. This variation thus allows reuse of sequences that are similar but not identical.

Brisk et al [23] also target the Alpha architecture and identify repeated phrases on a dataflow graph of intermediate code. Operating at an earlier compiler stage gives the compressor a higher-level view and more options. It naturally obligates later compiler phases to assign registers identically around each echo instruction, either by constraining the register assignments, 
by inserting spill code, or by abandoning echo instructions rendered uneconomical by the spill code, but the benefit can outweigh the cost.

Wu et al [24] target the IA32 instruction set and report that echo instructions make IA32 code roughly size-competitive with the THUMB extension to the ARM instruction set but with a lower performance overhead. They also propose a base register for echo instructions, in order to shorten long displacements.

Another natural implementation for echo instructions would be as part of a dynamic translator such as that in Transmeta's Crusoe architecture [26] or even using DISE [27] to replace echo instructions during decoding.

\section{Related work}

The literature on code compression has grown rapidly in the last few years [28], but much of it assumes a decompression step (perhaps into a hidden cache) and thus can use techniques that are incompatible with typical bytecode (and machinecode) interpreters, such as a front-to-back scan over the entire input. This section thus confines itself to representatives of the methods applicable in this scenario, namely dictionary-based compression that is compatible with direct interpretation.

Liao, Devadas, and Keutzer describe an instruction that appears identical to the echo instruction, but its interpretation differs in one important way, with the result that the two instructions are normally used quite differently [29, 30]. The instruction

CALD offset, len

has the same signature as the (basic) echo instruction, and it directs the processor to execute len instructions at address offset, but it fetches those instructions from a separate "dictionary" memory, not the one that holds the CALD and the main instruction stream. This seemingly modest semantic difference promotes very different styles of use:

- CALD's separate dictionary allows reordering to exploit overlap. For example, if one fragment is the triple A-B-C, and another is B-C-D, then the optimizer can store the sequence A-B-C-D in the dictionary, and two CALD's can share the single copy of B-C. Balancing this advantage is the cost of the separate dictionary memory. A set-covering algorithm identifies the sequences to put in the dictionary and exploits overlap. Also, CALD was designed for use in embedded systems, which could put the dictionary in ROM, which might be cheaper, larger, faster, or more sparing with power than the main program memory.

- Echo instructions make the symmetric trade-off. They take their fragments from code that's already present in the main memory and thus incur no cost for the dictionary memory, but the method for introducing echo instructions does not synthesize sequences - such as the A-B-C-D above - that appear nowhere in the original program and thus can neither benefit from the set-covering method nor easily split the program between ROM and another type of memory.

It is possible to use echo instructions unchanged with CALD-style dictionaries. One or more such dictionaries could be built and placed at a point in main memory where they can be executed only via echo instructions and never by having control fall into a synthetic sequence. For example, placing dictionaries after unconditional jumps would be safe. In this way, echo instructions could exploit overlapping like CALD instructions do, but also still reuse undictionaried phrases that are too long, appear too few times, or exploit too little overlap to justify inclusion in a dictionary. Such dictionaries for echo instructions would remain, however, in main memory, not ROM, so the two methods—despite all their similarities—still suit different needs.

Compression ratios ranging from 0.681-0.919x have been reported for the CALD instruction [30]. These numbers are, however, not directly comparable to those for the echo instruction above, for a variety of reasons. Chief among these is the fact that CALD was applied to optimized machine code and the echo instruction to unoptimized bytecode, which is probably more easily compressed.

Other dictionary-based code compressors [9, 10, 31, 32, 33, 34] associate a fixed code with the most common sequences in either the program at hand or a training set that is used to generate a code intended to suit all programs. This approach can be particularly advantageous on short programs and at the beginning of all programs, where the echo compressor has little prior context to exploit. On the other hand, a fixed code is naturally less effective when the program at hand is not well represented by the test suite or, in the case of per-program dictionaries, by a single, typically small dictionary. An advantage of LZ compression is that it effectively changes the dictionary as it progresses through the input. It seems likely that some inputs suit a fixed dictionary and others suit the changing dictionary offered by LZ77's sliding window. Thus neither approach seems likely to dominate, and the code compression community benefits from a menu that includes both methods. 
A complementary alternative is Huffman compression, which saves space by assigning short codes to common symbols in the alphabet. Conventional decompressors for Huffman codes require decoding the input bit by bit, which is less problematic in a conventional one-pass decompressor than in a bytecode interpreter, because interpreters necessarily decode loop bodies over and over. Recent work [35], however, shows a block decoder specialized for Huffman-coded instruction sets. It adds as little as $10-20 \%$ to interpretation time, and the interpreters are compact. It would be natural to combine this method with the echo instruction, substituting statistically rigorous Huffman codes for the ad hoc, manually designed encoding above. The echo instructions would exploit redundant sequences, and the Huffman coding would exploit the fact that some individual instructions merit shorter encodings. Combining the two methods ought to result in even better compression than either method in isolation.

\section{Acknowledgments}

This work benefited from discussions with Jack Davidson, Will Evans, Dave Hanson, Todd Proebsting and Kevin Scott.

\section{References}

1. B. Abali, H. Franke, D. Poff, and T. Smith. Performance of hardware compressed main memory. Research Report RC21799, IBM T. J. Watson Research Center, 7/2000.

2. C. Benveniste, P. Franaszek, and J. Robinson. Cache-memory interfaces in compressed memory systems. Research Report RC21662, IBM T. J. Watson Research Center, 2/2000.

3. D. Ditzel, H. McClellan, and A. Berenbaum. The hardware architecture of the CRISP microprocessor. Proceedings of the International Symposium on Computer Architecture:309-319, 6/1987.

4. M. Game and A. Booker. CodePack: Code compression for PowerPC processors. Technical report, IBM Microelectronics Division, 5/2000.

5. S. Larin and T. Conte. Compiler-driven cached code compression schemes for embedded ILP processors. Proceedings of the 32nd International Symposium on Microarchitecture:82-92, 11/1999.

6. C. Lefurgy, E. Piccininni, and T. Mudge. Analysis of a high performance code compression method. Proceedings of the 32nd International Symposium on Microarchitecture:93-102, 11/1999.

7. C. Lefurgy, E. Piccininni, and T. Mudge. Reducing code size with run-time decompression. Proceedings of the 6th International Symposium on High-Performance Computer Architecture:218-227, 1/2000.

8. S. Debray, W. Evans, R. Muth, and B. de Sutter. Compiler techniques for code compaction. TOPLAS 22(2):378-415, $3 / 2000$

9. W. Evans and C. Fraser. Bytecode compression via profiled grammar rewriting. PLDI’01:148-155, 6/2001.

10. T. A. Proebsting. Optimizing an ANSI C interpreter with superoperators. POPL'95:322-332, 1/1995.

11. J. Ziv and A. Lempel. A universal algorithm for sequential data compression. IEEE Transactions on Information Theory 23:337-342, 1977.

12. T. Bell, J. Cleary, and I. Witten. Text Compression. Prentice Hall, 1990.

13. P. Fenwick. Ziv-Lempel encoding with multi-bit flags. DCC’93:138-147, 1993.

14. K. Cooper and N. McIntosh. Enhanced code compression for embedded RISC processors. PLDI'99:139-149, 5/1999.

15. C. Fraser, E. Myers, and A. Wendt. Analyzing and compressing assembly code. Proceedings of the SIGPLAN'84 Symposium on Compiler Construction:117-121, 6/1984.

16. K. Kunchithapadam and J. Larus. Using lightweight procedures to improve instruction cache performance. Computer Sciences Technical Report \#1390, University of Wisconsin-Madison, 1/1999.

17. C. Fraser and D. Hanson. A Retargetable C Compiler: Design and Implementation. Addison Wesley Longman, 1995.

18. B. P. Tunstall. Synthesis of noiseless compression codes. Ph.D. thesis, Georgia Institute of Technology, 1967.

19. D. Huffman. A method for the construction of minimum redundancy codes. Proceeding. of IRE 40:1098-1101, 1952.

20. M. Adler and J.-l. Gailly. The gzip home page. http://www.gzip.org. 
21. C. Fraser. An instruction for direct interpretation of LZ77-compressed programs. Technical report MSR-TR-2002-90, 9/2002.

22. J. Lau, S. Schoenmackers, T. Sherwood, and B. Calder. Reducing code size with echo instructions. International Conference on Compilers, Architecture, and Synthesis for Embedded Systems:84-94, 10/2003.

23. P. Brisk, A. Nehapetian, and M. Sarrafzadej. Instruction Selection for Compilers that Target Architectures with Echo Instructions. 8th International Workshop on Software and Compilers for Embedded Systems (SCOPES), 9/2004.

24. Y. Wu, M. Breternitz, H. Hum, R. Peri, and J. Pickett. Echo Techology (ET) for Memory Constrained CISC Processors. Workshop on Compilers and Tools for Constrained Embedded Systems (CTCES), 9/2004.

25. D. Burger and T. Austin. The SimpleScalar tool set, version 2.0. Computer Architecture News 25(3):13-25, 6/1997.

26. A. Klaiber. The technology behind Crusoe processors. Transmeta Corporation, 2000. http://www.transmeta.com/about/press/white_papers.html.

27. M. Corliss, E. Lewis, and A. Roth. A DISE implementation of dynamic code decompression, LCTES:232-243, 6/2003.

28. R. van de Wiel. Code compaction bibliography. 2/2004. http://www.extra.research.philips.com/ccb/.

29. S. Liao. Code generation and optimization for embedded digital signal processors. PhD dissertation, MIT, 1996.

30. S. Liao, S. Devadas, and K. Keutzer. A text-compression-based method for code size minimization in embedded systems. TODAES 4(1):12-38, 1999.

31. P. Bird and T. Mudge. An instruction stream compression technique. Technical Report CSE-TR-319-96, EECS Department, University of Michigan, 11/1996.

32. J. Ernst, W. Evans, C. Fraser, S. Lucco, and T. Proebsting. Code compression. PLDI’97:358-365, 6/1997.

33. C. Lefurgy, P. Bird, I. Chen, and T. Mudge. Improving code density using compression techniques. Proceedings of the 30th International Symposium on Microarchitecture:194-203, 12/1997.

34. S. Lucco. Split-stream dictionary program compression. PLDI’00:27-34, 6/2000.

35. M. Latendresse and M. Feeley. Generation of fast interpreters for Huffman compressed bytecode. Proceedings of the Workshop on Interpreters, Virtual Machines and Emulators, 6/2003.

\section{Appendix 1: $C$ code to interpret an echo instruction}

The implementation of the echo instruction is part of a conventional bytecode interpreter. A central routine is interpret1, which accepts a single bytecode and a pointer to a structure that records the state of the interpreter, with fields holding the stack, the program counter, and so on. interpret1 performs a switch on the bytecode, and the case for the echo instruction simply calls on a procedure:

\section{case ECHO: \{doEcho(istate); break; \}}

This sample code interprets a basic echo instruction with a one-byte length and a two-byte displacement. Comments describe the process the line-by-line:

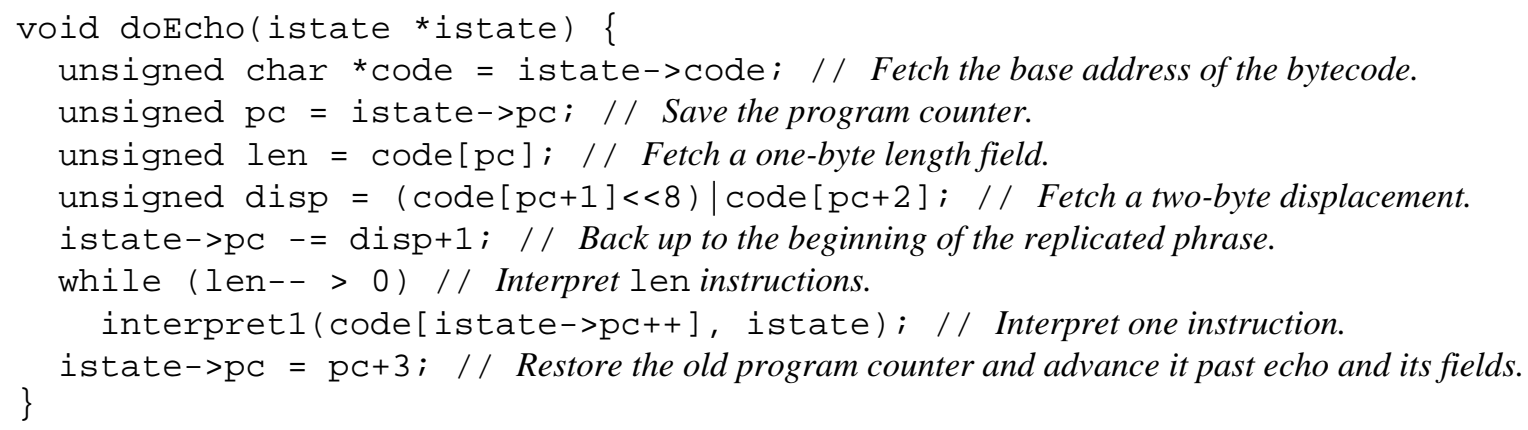




\section{Appendix 2: Instruction set}

The table below describes the un-typed or generic operators from the initial instruction set. A superscript denotes the number of literal bytes, if any, after the operator. The only changes from lcc [17] are literals, the LocalCALL operators (which use literals instead of the stack to identify the callee), and comparisons. lcc comparisons accept two comparands and a literal branch address, but the comparisons here accept two comparands and push a flag for BrTrue, which accepts the literal branch address.

The type suffixes are: $V$ for void or no value, $C$ and $S$ for char and short, $I$ and $U$ for signed and unsigned integers, $F$ and $D$ for single- and double-precision floating-point numbers, $\mathrm{P}$ for pointers, and $\mathrm{B}$ for instructions that operate on blocks of memory.

\begin{tabular}{|c|c|}
\hline Operator & Comment \\
\hline ADD DIV MOD MUL SUB & Arithmetic. \\
\hline BAND BOR BXOR & Bit-wise Booleans. \\
\hline $\mathrm{BCOM}$ & Bit-wise negation. \\
\hline NEG & Arithmetic negation. \\
\hline CVD & Convert from double, float, int. \\
\hline CVI1 CVI2 & Sign-extend char, short. \\
\hline CVU1 CVU2 & Zero-extend char, short. \\
\hline EQ GE GT LE LT NE & Compare and push 0 or 1. \\
\hline LSH RSH & Shifts. \\
\hline INDIR & Pop p, push *p. \\
\hline ASGN & Pop p and v, copy v to *p. \\
\hline $\mathrm{ASGNB}^{2}$ & Pop p and v, copy the block at *v to *p. \\
\hline $\mathrm{ADDRF}^{2} \mathrm{ADDRG}^{2} \mathrm{ADDRL}^{2}$ & Push address of formal, global, or local. \\
\hline JUMP $^{2}$ & Pop label number, jump. \\
\hline ARG & Top is next outgoing argument. \\
\hline RET & Return value atop stack. \\
\hline CALL & Pop p, call routine at address p. \\
\hline LocalCALL $^{2}$ & Call routine at literal address. \\
\hline POP & Discard top element. \\
\hline LIT1 $^{1}$ LIT2 $^{2}$ LIT3 $^{3}$ LIT $^{4}$ & Push 1-4 literal bytes. \\
\hline BrTrue $^{2}$ & Pop flag. Jump if true. \\
\hline
\end{tabular}


Figure 1. Distribution of lengths

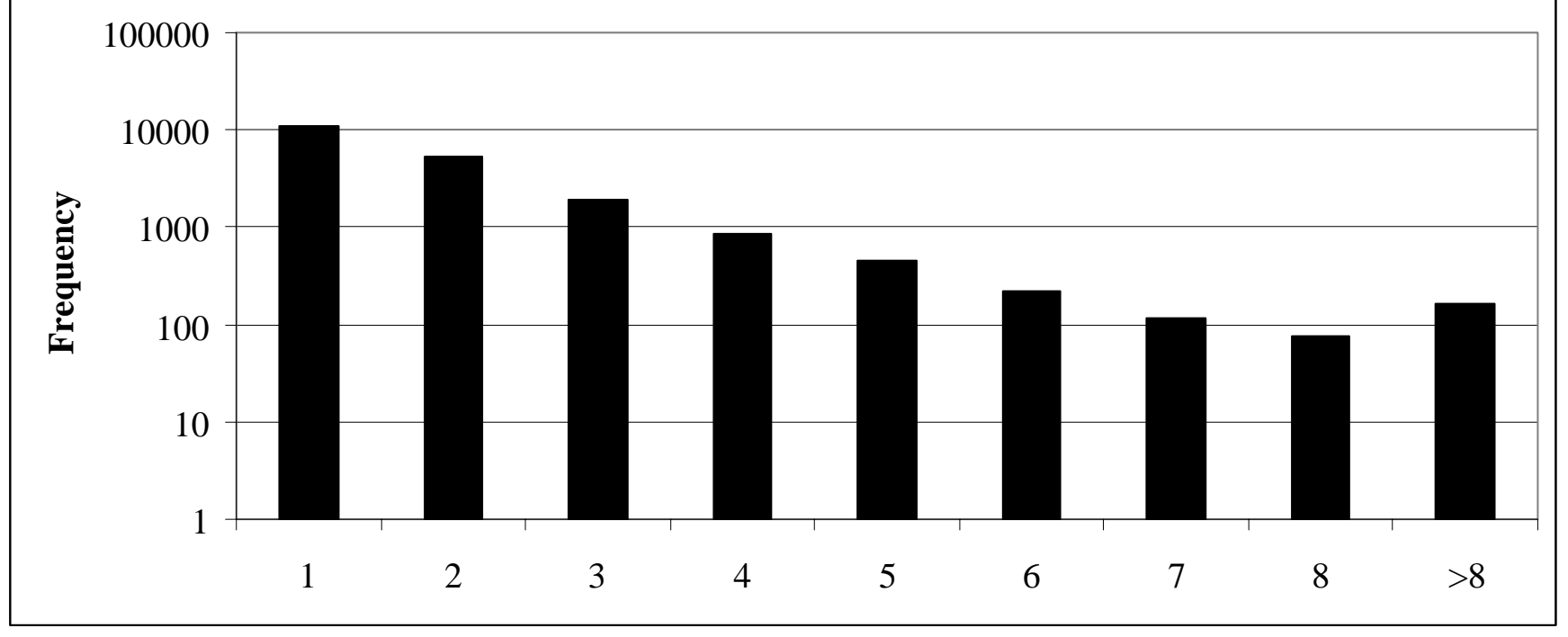




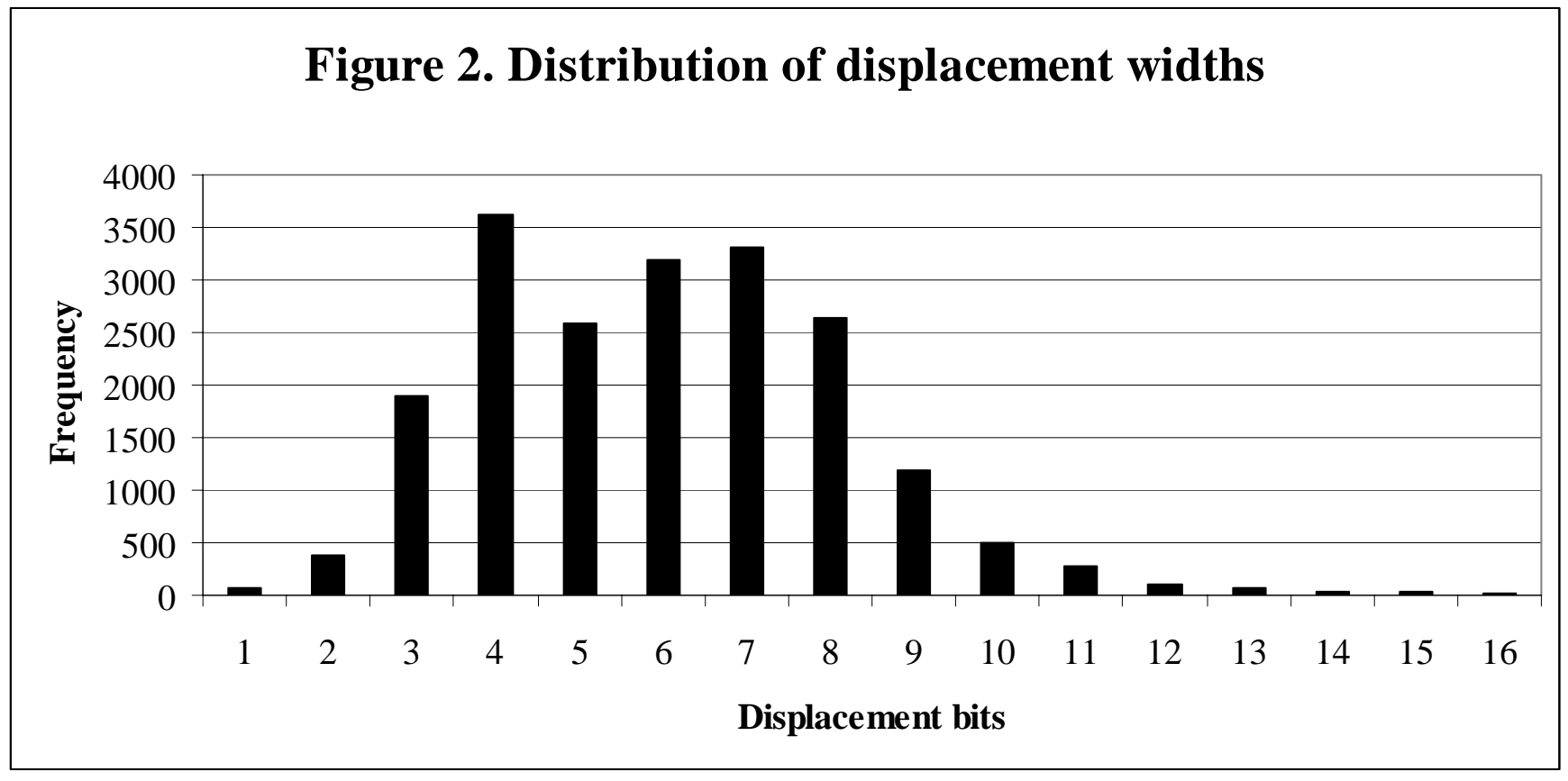




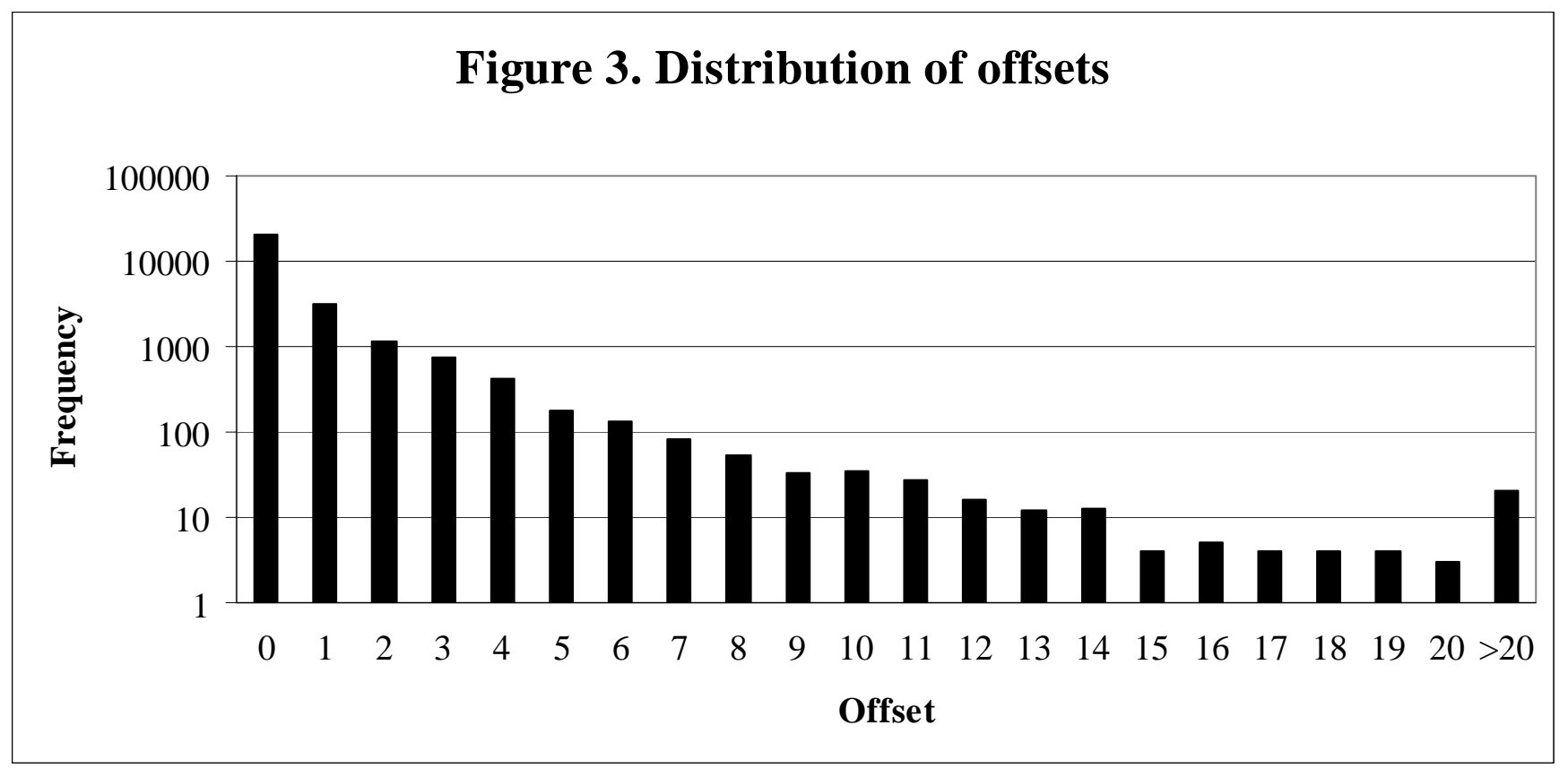

